\title{
Adjuvant external beam radiation and brachytherapy for vaginal resection margin positive cervical cancer
}

\author{
Donghyun Kim, MD, PhD', Yongkan Ki, MD, PhD², Wontaek Kim, MD, PhD', \\ Dahl Park, PhD', Joohye Lee, MD², Jayoung Lee, MD, PhD², \\ Hosang Jeon, $\mathrm{PhD}^{2}$, Jiho Nam, MD \\ 'Department of Radiation Oncology and Biomedical Research Institute, Pusan National University Hospital, \\ Pusan National University School of Medicine, Busan; \\ ${ }^{2}$ Department of Radiation Oncology, Pusan National University Yangsan Hospital, \\ Pusan National University School of Medicine, Yangsan, Korea
}

Purpose: To evaluate the treatment outcomes of adjuvant external beam radiation therapy (EBRT) and vaginal brachytherapy (VB) following radical hysterectomy in cervical cancer patients with involved vaginal resection margin (VRM).

Materials and Methods: We retrospectively reviewed the medical records of 21 patients treated with postoperative EBRT and VB for positive VRM FIGO stage IB-IIA cervical cancer between 2003 and 2015. Concurrent platinum-based chemotherapy was administered to all patients.

Results: The median whole pelvis EBRT dose was $50.4 \mathrm{~Gy}$ (range, 45 to $50.4 \mathrm{~Gy}$ ). In the VB, the median dose per fraction, number of fractions, and total dose delivered were: $4 \mathrm{~Gy}$ (range, 3.0 to $4.0 \mathrm{~Gy}$ ), 4 fractions (range, 3 to 5 fractions), and 16 Gy (range, 12 to $20 \mathrm{~Gy}$ ), respectively. At a median follow-up of 46 months (range, 9 to 122 months), local recurrence was observed in 2 patients, and distant metastasis was present in 7 patients. All patients with local recurrence subsequently developed distant metastases. The 5-year local control, disease-free survival, and overall survival rates were $89.1 \%, 65.9 \%$, and $62.9 \%$, respectively. Of the 21 patients, 7 patients (33.3\%) reported grade 2 acute toxicity; however, there were no grade 3 or higher acute adverse events. Grade 1-2 late toxicities were observed in 8 patients. Late grade 3 urinary toxicity was reported in 1 patient.

Conclusions: Adjuvant EBRT and VB showed excellent local control and low toxicity in cervical cancer patients with positive VRM. Although limited by its retrospective nature, the findings from our study provide evidence supporting the use of additional VB in pathologically involved VRM.

Keywords: Uterine cervical cancer, Brachytherapy, Positive surgical margin, Vaginal recurrence

\section{Introduction}

Sometimes adjuvant vaginal brachytherapy (VB) is employed after external beam radiation therapy (EBRT) in the management of post-hysterectomy cervical cancer. Brachytherapy enables to deliver a localized radiation dose to the vaginal cuff after hysterectomy [1]. Several studies have shown that the use of $\mathrm{VB}$ reduce local recurrence in the region

Received 27 February 2018, Revised 06 April 2018, Accepted 03 May 2018.

Correspondence: Yongkan Ki, MD, PhD, Department of Radiation Oncology, Pusan National University Yangsan Hospital, Pusan National University School of Medicine, Yangsan 50612, Korea. Tel: +82-51-240-7383, Fax: +82-51-240-7457, E-mail: apex7171@hanmail.net

(c) This is an Open Access article distributed under the terms of the Creative Commons Attribution Non-Commercial License (http://creativecommons.org/ licenses/by-nc/4.0/) which permits unrestricted non-commercial use, distribution, and reproduction in any medium, provided the original work is properly cited.

www.e-roj.org 
of the vaginal cuff in endometrial cancer [2-4]. In contrast to the more abundant data for brachytherapy in endometrial cancer, the study of VB after EBRT for cervical cancer has been lacking. Therefore, there is no clear agreement as to the indications for VB after EBRT for cervical cancer.

The American Brachytherapy Society consensus guidelines [5] suggest that VB may be used after EBRT in postoperative patients with high risk factors, such as close or positive margins, less than radical hysterectomy $(\mathrm{RH})$, large or deeply invasive tumors, parametrial or vaginal involvement, or extensive lymphovascular invasion. According to the National Comprehensive Cancer Network cervical cancer guidelines [6], patients with positive or close vaginal resection margin (VRM) after surgical treatment, additional VB should be considered. If the VRM is positive, additional VB with a dose range of 10-15 Gy is an option; however, there is no supporting evidence for this recommendation.

The oncologic outcomes of additional VB have yet to be established. The purpose of this study is to report our clinical experience with adjuvant EBRT and high-dose-rate (HDR) VB in VRM positive cervical cancer patients after RH.

\section{Materials and Methods}

\section{Patients and tumor characteristics}

Between 2003 and 2015, 24 patients underwent RH and bilateral salpingo-oophorectomy with pelvic lymphadenectomy followed by postoperative EBRT and VB for VRM positive cervical cancer. Three patients with positive parametrial margin were excluded and a total of 21 patients were included in the analysis. All patients were staged according to the 2009 International Federation of Gynecology and Obstetrics (FIGO) staging criteria [7]. Adverse events (AEs) were graded according to the Common Terminology Criteria for Adverse Events (CTCAE) version 4.0 [8]. The grade of the AE describes the maximum level of toxicity identified during follow-up. Toxicities that occurred more than 90 days after the completion of VB were considered as late toxicities. The Institutional Review Board of Pusan National University Hospital reviewed the protocol and exempted this study from further review (No. H-1801-018063).

\section{Treatment}

All patients underwent $\mathrm{RH}$, bilateral salpingo-oophorectomy and pelvic lymph node (PLN) dissection. Para-aortic lymph node was sampled in 14 patients (66.7\%) with suspicion of metastases. All patients received a median of 6 cycles (range, 3 to 6 cycles) concurrent weekly cisplatin $\left(40 \mathrm{mg} / \mathrm{m}^{2}\right)$ chemotherapy. Consolidation chemotherapy with cisplatinpaclitaxel combination regimen (range, 2 to 5 cycles) was administered to 5 patients (23.8\%) with multiple PLN metastasis. Neoadjuvant chemotherapy with cisplatin and paclitaxel (range, 2 to 3 cycles) was used to 5 patients.

Adjuvant whole-pelvis EBRT was initiated within 4-6 weeks after surgery. EBRT was delivered with a 15-MV X-ray from a linear accelerator. A total dose of 45.0-50.4 Gy was administered over 5-6 weeks, five times per week with $1.8 \mathrm{~Gy}$ per fraction. The superior margin of the whole pelvis field was extended to the L4-5 interspace. The inferior margin was the inferior border of the obturator foramen, and laterally, the field extended at least $1 \mathrm{~cm}$ beyond the lateral margin of the bony pelvic wall. The lateral portal anterior margin was placed at the pubic symphysis, and the posterior margin was at the S2-S3 interspace.

VB was delivered with an Ir-192 brachytherapy unit (microSelectron; Nucletron, Veenendaal, The Netherlands), after 39.6-45.0 Gy of EBRT. Single channel vaginal cylinder (diameter ranged from 3.0 to $3.5 \mathrm{~cm}$ ) was used for post-hysterectomy adjuvant brachytherapy of the vaginal cuff. All patients were instructed to empty their bladders before insertion of a vaginal cylinder. A rectal catheter was used to inject $30 \mathrm{~mL}$ of diluted contrast. Condoms were placed over the cylinders when they are inserted into the vagina to facilitate cleaning and preparation. Xylocaine jelly was applied to lubricate the applicator. After the cylinder applicator was positioned, the applicator was locked in place with a base board, which was immobilized underneath the patient. For three-dimensional (3D) planning, all patients underwent computed tomography (CT) scan with 2.0-mm slice thickness to improve the image resolution for digitizing the source positions. All CT slices were transferred to the 3D treatment planning system Oncentra Brachy (Nucletron). The Oncentra Brachy planning system uses the Task Group No. 43 Report dose calculation formalism. A dose of 3-4 Gy was prescribed to a depth of $0.5 \mathrm{~cm}$ from the surface of the applicator and the treatment length was the upper $3 \mathrm{~cm}$ of the vagina (Fig. 1). The dose received by 2 $\mathrm{mL}(\mathrm{D} 2 \mathrm{~mL})$ of rectum and bladder volume was determined to assess any possible relationship with the treatment-induced toxicity. It was given twice weekly with at least 48 hours between fractions. No dose point optimization was performed. Portable X-ray units were used to verify the applicator position in relation to the pelvic bony structures. On completion of the course of treatment, patients were educated on the use of vaginal dilators and they were instructed to use them two 


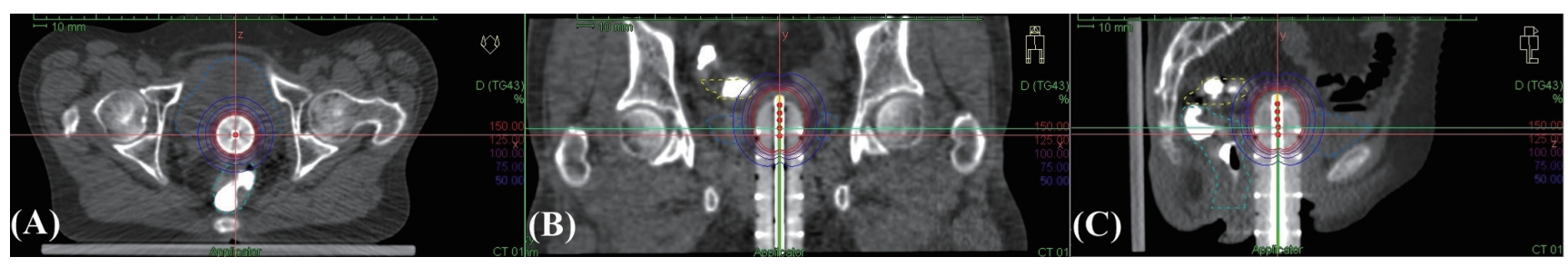

Fig. 1. Example of isodose distribution in the axial (A), coronal (B), and sagittal (C) planes.

times per week beginning 2 weeks after treatment.

\section{Statistical methods}

Continuous variables were analyzed using the Student t-test and two-tailed p-values less than 0.05 were considered statistically significant. Frequencies were compared in all patients using the Fisher exact test for categorical variables. We estimated local control (LC), distant failure, and the incidence of treatment-related toxicity using cumulative incidence functions. Local recurrence was defined by either pathologic exam or an imaging study showing regrowth of tumor on the vaginal stump. Regional recurrence was defined by a new finding of metastatic PLN or pelvic side wall, and distant metastasis was defined as metastases in distant lymph node including para-aortic lymph node or organs outside the pelvic cavity. The time to recurrence was defined from the date of surgery to the date of first recurrence. Disease-free survival (DFS) was defined as the time between hysterectomy and the first recurrence of the disease or the last follow-up. Overall survival (OS) was defined as the time between hysterectomy and death from any cause or the last follow-up. Survival curves were generated using the Kaplan-Meier method and differences were tested by use of the log-rank test. The PLN metastasis and parametrial invasion were analyzed whether these factors have a prognostic value. All statistical analyses were performed using the SPSS version 18.0 software (SPSS Inc., Chicago, IL, USA).

\section{Results}

Patient and tumor characteristics are summarized in Table 1. The median age was 48 years (range, 35 to 72 years). The majority of patients (17 of 21 patients, 81.0\%) were FIGO stage IB. The most common histopathologic type was squamous cell carcinoma (17 patients, 81.0\%). The median tumor size in the hysterectomy specimens was $4.8 \mathrm{~cm}$. Parametrial and PLN involvement was present in $23.8 \%$ and $61.9 \%$ of the patients, respectively. The mean time from surgery to the start of EBRT was 5.1 weeks (range, 4.2 to 5.9 weeks). The median EBRT dose was 50.4 Gy (range, 45 to 50.4 Gy) to the whole pelvis and midline block was not used. Concurrent chemoradiotherapy was well tolerated, with 1 case (4.8\%) of treatment interruption (7 days). The median dose per fraction, number of fractions, and total dose for VB were $4 \mathrm{~Gy}$ (range, 3.0 to $4.0 \mathrm{~Gy}$ ), 4 fractions (range, 3 to 5), and 16 Gy (range, 12 to $20 \mathrm{~Gy}$ ), respectively. The median equivalent dose in $2 \mathrm{~Gy} /$ fraction (EQD2) [9] of EBRT + VB was 68.2 Gy (range, 63.6 to

Table 1. Clinical and pathologic characteristics $(n=21)$

\begin{tabular}{lc}
\hline \multicolumn{1}{c}{ Characteristic } & Value \\
\hline Age (yr) & $48(35-72)$ \\
Tumor size in the surgical specimen (cm) & $4.8(2.6-8.5)$ \\
Histologic type & \\
Squamous cell carcinoma & $17(81.0)$ \\
Adenocarcinoma & $4(19.0)$ \\
Histologic grade & \\
Well differentiated & $2(9.5)$ \\
Moderately differentiated & $14(66.7)$ \\
Poorly differentiated & $5(23.8)$ \\
FIG0 stage & \\
IB1 & $8(38.1)$ \\
IB2 & $9(42.9)$ \\
IIA1 & $2(9.5)$ \\
IIA2 & $2(9.5)$ \\
Depth of invasion & \\
$<2 / 3$ & $2(9.5)$ \\
$\geq 2 / 3$ & $19(90.5)$ \\
LVSI & \\
Negative & $10(47.6)$ \\
Positive & $11(52.4)$ \\
Parametrial involvement & \\
Negative & $16(76.2)$ \\
Positive & $5(23.8)$ \\
Lymph node involvement & \\
Pogative & \\
\hline
\end{tabular}

Values are presented as median (range) or number of patients (\%). FIGO, International Federation of Gynecology and Obstetrics; LVSI, lymphovascular space invasion. 


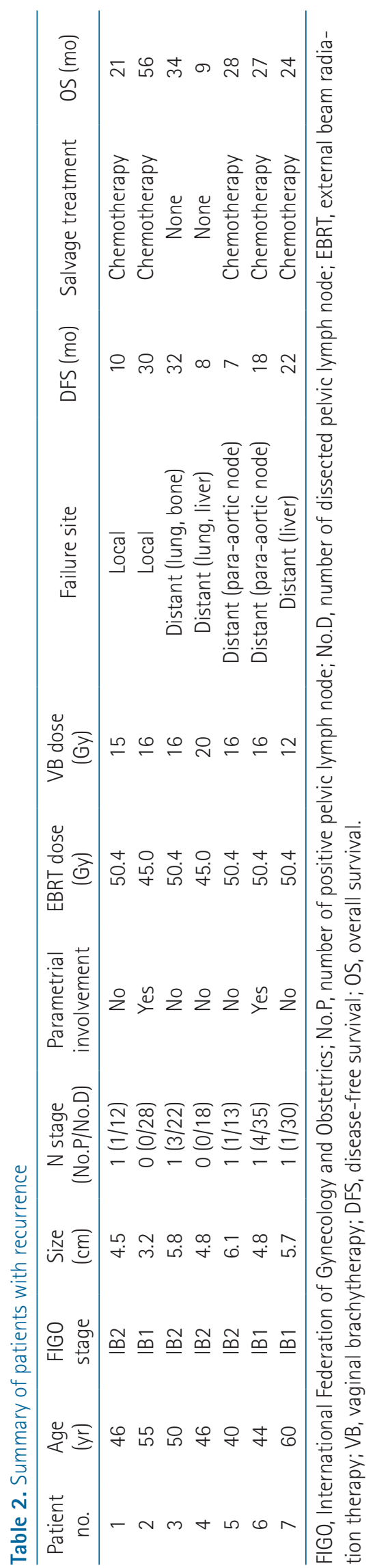

150 www.e-roj.org
72.9 Gy).

The median follow-up period was 46 months (range, 9 to 122 months). Seven patients (33.3\%) experienced treatment failures after completion of treatment and all of these patients had died at the time of analysis. Local failure occurred in 2 patients, while regional failure and distant metastasis occurred in 0 and 7 patients, respectively. Of the 7 patients presenting with disease progression, salvage chemotherapy was performed in 5 patients. All patients with local recurrence developed subsequent distant metastases. The details of patients who experienced recurrences are summarized in Table 2. As shown in Fig. 2, the 5-year LC, DFS, and OS rate were $89.1 \%, 65.9 \%$, and $62.9 \%$, respectively.

The difference of mean EBRT + VB EQD2 between local recurrence and non-local recurrence group was not statistically significant (69.2 Gy vs. $68.0 \mathrm{~Gy} ; \mathrm{p}=0.517$ ). The pathologic tumor size was also not significantly different between local recurrence and non-local recurrence group $(3.8 \pm 0.9 \mathrm{~cm}$ and $5.2 \pm 1.6 \mathrm{~cm} ; p=0.262)$. There was no relationship between PLN metastasis $(p=0.629)$ and recurrence, or parametrial invasion $(p=0.429)$ and recurrence. The 5 -year DFS was not significantly different with regard to PLN metastasis $(75.0 \%$ vs $60.6 \% ; p=0.525)$, or parametrial invasion (61.9\% vs. $80.0 \%$; $p$ $=0.458)$, respectively.

The median D2mL (EQD2) for rectum and bladder were 65.8 Gy (range, 62.2 to $70.6 \mathrm{~Gy}$ ) and $70.8 \mathrm{~Gy}$ (range, 65.6 to 75.4 Gy), respectively. A summary of AEs reported can be seen in Table 3. We observed grade 2 acute treatment-related toxicities in 7 of 21 patients. Grade 2 acute AEs consisted of fatigue ( $n=$ 4), nausea ( $n=3)$, constipation ( $n=2)$, and urinary frequency/ urgency $(n=1)$. There was no grade 3 or higher acute AE. Late toxicities occurred in 8 out of 21 patients. Cystitis was the most common late toxicities, and was seen in 7 patients (33.3\%). One patient with the EQD2 of D2mL bladder is $72.6 \mathrm{~Gy}$ experienced grade 3 cystitis (gross hematuria) 14 months after treatment. This patient was managed conservatively, and the symptoms subsided at last follow-up. No grade 4 late toxicity was seen

\section{Discussion and Conclusion}

HDR VB is an important component of treatment for gynecologic malignancies. A positive resection margin, which means that cancer cells may remain in the operation bed is an important risk factor for local recurrence. Therefore VB after EBRT to improve LC needs to be considered. In our study, high locoregional control was achieved through adjuvant EBRT and 
A

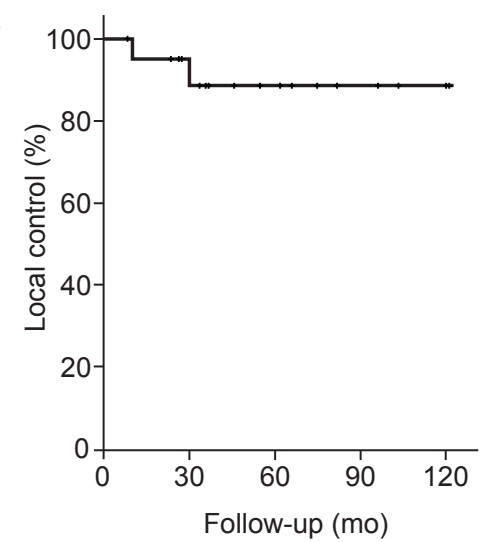

B

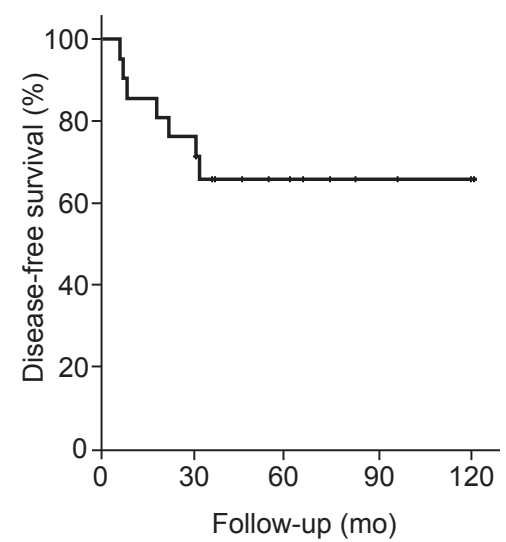

C

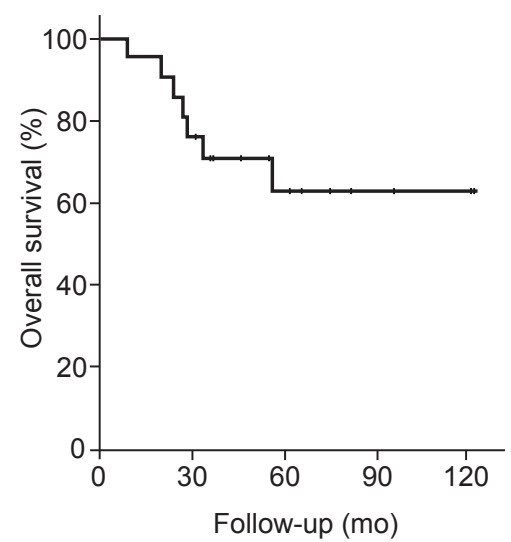

Fig. 2. Kaplan-Meier local control (A), disease-free survival (B), and overall survival (C) curves of all patients.

Table 3. Treatment induced adverse events

\begin{tabular}{lcccc}
\hline \multicolumn{1}{c}{ Adverse event } & Grade & Grade & Grade & Total \\
& 1 & 2 & 3 & \\
\hline Acute toxicities & & & & \\
$\quad$ Fatigue & 10 & 4 & 0 & 14 \\
$\quad$ Nausea & 8 & 3 & 0 & 11 \\
$\quad$ Vomiting & 2 & 0 & 0 & 2 \\
$\quad$ Diarrhea & 3 & 0 & 0 & 3 \\
$\quad$ Constipation & 2 & 2 & 0 & 4 \\
$\quad$ Urinary frequency/urgency & 3 & 1 & 0 & 4 \\
Late toxicities & & & & \\
$\quad$ Cystitis & 4 & 2 & 1 & 7 \\
Proctitis & 2 & 0 & 0 & 2 \\
$\quad$ leus & 0 & 1 & 0 & 1 \\
\hline
\end{tabular}

Values are presented as number.

VB following surgery in VRM positive cervical cancer. Distant metastasis comprised the majority of treatment failures. This findings are in agreement with previous studies that evaluated for endometrial cancer [2-4]. In addition, we showed that treatment related toxicity was minimal.

A positive VRM is an uncommon result for gynecologic surgeons, and the numbers of patients with positive VRM are too few to conduct randomized trials to test the efficacy of additional boost. There are few publications examining the clinical outcome of VRM positive cervical cancer patients. Huh et al. [10] investigated the role of postoperative EBRT and VB among the 48 patients with positive VRM. The pelvic recurrence and distant metastasis rates were $8 \%$ and $15 \%$, respectively. The recurrence rate was 21\% (9/43) in those treated with EBRT and VB, and 40\% (2/5) in the EBRT-only treated group. Although very few data are available for comparison, our treatment result was comparable to those cited in the literature.

Averette et al. [11] reported that the 5-year OS rate was $50.8 \%$ in 61 patients with involved VRM after RH alone, and that the incidence of positive VRM was $4 \%$ in stage IB disease, increasing to $20 \%$ in stage IIA lesions. The 5 -year OS and recurrence-free survival rate was $82.8 \%$ and $78.7 \%$ for 25 patients with VRM positive cervical cancer treated with postoperative EBRT followed by VB without concurrent chemotherapy [12]. In the current study, 5-year OS and DFS rates were $62.9 \%$ and $65.9 \%$. However, the patients characteristics were not described in detail and the treatment results could not be compared. For close VRM (less than $5 \mathrm{~mm}$ ) cervical cancer patients, adjuvant EBRT and VB increased the 5-year OS rate from 28.6\% to $81.3 \%$ [13]. Because of the small number of VRM involved patients who treated with adjuvant EBRT alone, a comparison between adjuvant EBRT alone and EBRT followed by VB was not conducted in this study.

Ampil et al. [14] reported severe complication rates of 5\% to $24 \%$ in patients with elective postoperative external pelvic irradiation and additional VB after RH. Atkovar et al. [15] reported 37 patients who had received EBRT (median, 50 Gy) and additional HDR-VB after surgery for clinical early stage carcinoma of the cervix. Total HDR-VB dose ranged from 10 to $30 \mathrm{~Gy}$ (8-10 Gy per fraction) in weekly intervals. Seven patients developed late complications, consisting of 2 mild and 2 moderate cases of urinary complications and 2 mild and 1 (recto-vaginal fistula) severe case of rectal complications.

In our study, we demonstrated that adjuvant EBRT and VB with concurrent chemotherapy was well tolerated with low incidence of acute and chronic toxicity. Most AEs were clinically acceptable and one hospitalization indicated severe complication developed. In late AEs, urinary complication 
was predominant, while there was only 1 grade 2 or higher gastrointestinal toxicity. Late radiation effects could occur especially when brachytherapy follows EBRT, and all attempts should be made to reduce these occurrences.

To our knowledge, there are few reports assessing the treatment outcome of VB after EBRT with respect to the LC and AEs for the VRM positive cervical cancer. However, our study also had several limitations. First, the small number of patients limits the generalization of the results. Second, tumor characteristics and chemotherapy application are inconsistent due to its retrospective design. Neoadjuvant and adjuvant chemotherapy may have affected pathologic characteristics and pattern of recurrence. Lastly, the difference in the efficacy of an EBRT alone versus EBRT followed by VB cannot be compared directly. Therefore, it was difficult to conclude that VB following EBRT can salvage the patients with positive VRM, and further research is warranted to investigate the impact of additional VB on LC.

In conclusion, our study suggests that acceptable oncologic outcomes are achievable with postoperative EBRT followed by VB for VRM involved cervical cancer patients without significant toxicities. If VRM is involved after $R H, V B$ after EBRT to increase the total dose to the vaginal cuff needs to be considered to improve $\mathrm{LC}$ rates.

\section{Conflict of Interest}

No potential conflict of interest relevant to this article was reported.

\section{Acknowledgments}

This work was supported by clinical research grant from Pusan National University Hospital in 2018.

\section{References}

1. Sevin BU, Nadji M, Lampe B, et al. Prognostic factors of early stage cervical cancer treated by radical hysterectomy. Cancer 1995;76(10 Suppl):1978-86.

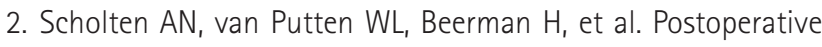
radiotherapy for Stage 1 endometrial carcinoma: longterm outcome of the randomized PORTEC trial with central pathology review. Int J Radiat Oncol Biol Phys 2005;63:834-8.

3. Alektiar KM, Venkatraman E, Chi DS, Barakat RR. Intravaginal brachytherapy alone for intermediate-risk endometrial cancer. Int J Radiat Oncol Biol Phys 2005;62:111-7.

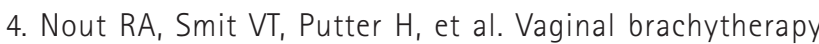
versus pelvic external beam radiotherapy for patients with endometrial cancer of high-intermediate risk (PORTEC-2): an open-label, non-inferiority, randomized trial. Lancet 2010;375:816-23.

5. Small W Jr, Beriwal S, Demanes DJ, et al. American Brachytherapy Society consensus guidelines for adjuvant vaginal cuff brachytherapy after hysterectomy. Brachytherapy 2012;11:58-67.

6. National Comprehensive Cancer Network. NCCN Clinical Practice Guidelines in Oncology (NCCN Guidelines) cervical cancer [Internet]. Fort Washington, PA: National Comprehensive Cancer Network; c2018 [cited 2018 Jun 1]. Available from: https://www.ncen.org/professionals/physician gls/default.aspx.

7. Pecorelli S. Revised FIGO staging for carcinoma of the vulva, cervix, and endometrium. Int J Gynaecol Obstet 2009;105:103-4.

8. National Cancer Institute. Common Terminology Criteria for Adverse Events (CTCAE) v4.0 and Common Toxicity Criteria (CTC) [Internet]. Bethesda, MD: National Institutes of Health c2018 [cited 2018 Jun 1]. Available from: http://ctep.cancer. gov/protocolDevelopment/electronic_applications/ctc.htm.

9. Nag S, Gupta N. A simple method of obtaining equivalent doses for use in HDR brachytherapy. Int J Radiat Oncol Biol Phys 2000;46:507-13.

10. Huh SJ, Kim WD, Ha SW, et al. Postoperative radiotherapy in carcinoma of the cervix with microscopically positive resection margin. Int J Clin Oncol 1997;2:147-51.

11. Averette $H E, N$ guyen $H N$, Donato DM, et al. Radical hysterectomy for invasive cervical cancer: a 25-year prospective experience with the Miami technique. Cancer 1993;71(4 Suppl):1422-37.

12. Lai $\mathrm{CH}$, Hong JH, Hsueh $\mathrm{S}$, et al. Preoperative prognostic variables and the impact of postoperative adjuvant therapy on the outcomes of stage IB or II cervical carcinoma patients with or without pelvic lymph node metastases: an analysis of 891 cases. Cancer 1999;85:1537-46.

13. Estape $R E$, Angioli $R$, Madrigal $M$, et al. Close vaginal margins as a prognostic factor after radical hysterectomy. Gynecol Oncol 1998;68:229-32.

14. Ampil F, Datta R, Datta S. Elective postoperative external radiotherapy after hysterectomy in early-stage carcinoma of the cervix. Is additional vaginal cuff irradiation necessary? Cancer 1987;60:280-8.

15. Atkovar G, Uzel O, Ozsahin M, et al. Postoperative radiotherapy in carcinoma of the cervix: treatment results and prognostic factors. Radiother Oncol 1995;35:198-205. 